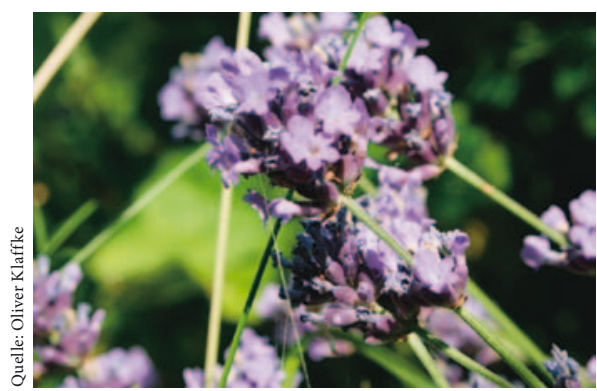

\section{Lavendel hilft bei Angststörungen}

Bei Angststörungen werden häufig Benzodiazepine eingesetzt; pflanzliche Mittel spielen bei der Behandlung bisher keine Rolle. Jetzt sind die Ergebnisse mehrerer Studien veröffentlicht worden, die sich mit der Rolle von Lavendelpräparaten bei der Therapie von Patienten beschäftigen, die unter Angstzuständen leiden. Lavendel wird bereits seit der Antike in der Medizin verwendet, und Extrakte finden als ätherische Öle, die etwa bei Demenzkranken eine beruhigende Wirkung haben, in der Aromatherapie Verwendung. Seit längerem wird auch vermutet, dass sich der Zustand von Menschen mit einer Angststörung verbessert, wenn sie Massagen mit Lavendelöl bekommen. In Untersuchungen wurde bereits vor 10 Jahren gezeigt, dass sich damit der Wert des Hamilton Anxiety Ratings senken und der Wert für die Stimmung steigern liess. In der Online-Vorausgabe der Zeitschrift International Clinical Psychopharmacology stellte Siegfried Kaspar von der Medizinischen Universität in Wien das Ergebnis einer Studie vor, die an 221 Patienten die Wirkung einer Therapie mit Lavendelkapseln untersuchte. Dabei zeigte sich, dass sich der Zustand von Probanden deutlich besserte, die das Lavendelpräparat statt eines Placebos einnahmen. Die Placebogruppe der Studie litt weiter an den typischen

\section{Ganzheitsmedizin aktuell}

Schweiz Z Ganzheitsmed 2010;22:254-255 DOI: 10.1159/000319542

Symptomen wie Sorgen, Schweissausbrüchen, Muskelverspannungen oder Angespanntsein. Dass Lavendelpräparate aber eine Alternative $\mathrm{zu}$ einer herkömmlichen medikamentösen Therapie sein können, zeigen die Ergebnisse einer multizentrischen Doppelblindstudie (Phytomedicine 2010;17:94-99), die vom Facharzt für Neurologie Helmut Woelk und Sandra Schläfke von der Wilmar Schwabe $\mathrm{GmbH}$ durchgeführt wurde. Sie verglichen Patienten, die mit dem Lavendelpräparat, einem Benzodiazepin und einem Placebo behandelt wurden. Dabei zeigte sich, dass die Wirkung der herkömmlichen Behandlung und mit Lavendel gleich wirksam war. Die Angstzustände gingen zurück. Überlegen war das Phytopräparat allerdings, was die Verträglichkeit betrifft. Es zeigte deutlich weniger Nebenwirkungen. Der Lavendelextrakt scheint Stoffe zu enthalten, die auf die $\mathrm{GABA}_{\mathrm{A}}$-Rezeptoren wirken. Neben der jetzt bestätigten anxiolytischen Wirkung gibt es auch Hinweise auf einen antidepressiven Effekt. (rfi)

\section{Orthomolekulare Therapie verbessert die Stimmung}

Mit Phenylalanin und Tryptophan stehen in der Orthomolekularen Therapie zwei Verbindungen zur Verfügung, um Patienten zu behandeln, die einer Stimmungsaufhellung bedürfen. Sie unterstützen den Körper beim Aufbau von Neurotransmittern, die von der Wissenschaft mit der Aufhellung der Stimmung in Verbindung gebracht werden. Phenylalanin ist ein Eiweissbestandteil, der bei der Botenstoffsynthese benötigt wird. Mit der täglichen Gabe von 0,5 bis $3 \mathrm{~g}$ wird der Körper beim Aufbau von Transmittern unterstützt. Essenziell ist dafür auch die Aminosäure Tryptophan, die ebenfalls benötigt wird und die mit der gleichen täglichen Dosierung von 0,5 bis 3 g gegeben werden sollte.

Zur Unterstützung des Nervensystems stehen in der Orthomolekularen Therapie auch noch verschiedene Vitamin-B-Komplexpräparate zur Verfügung, die einen hohen Stellenwert besitzen. Mit einer Dosierung von $50 \mathrm{mg}$ pro Tag sollten Patienten die Vitamine B1, B2, B3 und B6 nehmen. Das Vitamin B12 sollte einmal pro Woche intramuskulär gespritzt werden. Wichtig für die Stärkung des Nervensystems ist auch die Einnahme von 2 bis $6 \mathrm{~g}$ Vitamin $\mathrm{C}$ und von $600 \mathrm{mg}$ Magnesium täglich. (rfi)

\section{Ignatiusbohne gegen Hysterie}

Ignatia kann bei Frauen mit einem hysterischen oder nervösen Temperament zu einer Beruhigung und einer ausgeglichenen Stimmung führen. Mit der Potenzierung D3 bis D6 ist die Ignatiusbohne in diesen Fällen angezeigt. Bei den Patientinnen wechselt die Stimmung rasch. Es können Schweigsamkeit, Melancholie, Traurigkeit, Introversion, Depression oder unwillkürliches Schluchzen und Seufzen vorherrschen. Für die Lebensqualität ist es wichtig, den Kreislauf der belastenden Krisen zu durchbrechen. Die Auslöser dafür sind Kummer und Sorgen, die die Patientin belasten, oder eine momentane Überanstrengung und Aufregung, die sie an den Rand der Belastbarkeit bringen. (rfi)

\section{Kluge Eltern wollen pflanz- liche Mittel für ihr Kind}

Wenn ihre Kinder krank sind, wenden Eltern bevorzugt pflanzliche Arzneimittel an. Zu den eifrigsten Befürwor- 
tern der Phytopharmaka gehören vor allem Mütter und Väter mit einem höheren Bildungsstand, Eltern, die in einer Stadt leben, und Familien, die über ein höheres Einkommen verfügen. Das ergab eine aktuelle Studie an zwei Universitätskinderkliniken in Leipzig und München sowie in zwei Leipziger Kinderarzt-Praxen. Der Leipziger Kinder- und Jugendarzt Prof. Dr. Wieland Kiess und sein Team befragten mit einem standardisierten Fragebogen die Eltern von insgesamt 413 Kindern zum Einsatz und Behandlungserfolg pflanzlicher Arzneimittel. Sie veröffentlichten ihre Ergebnisse in der Deutschen Medizinischen Wochenschrift (2010;135:959964). Besonders bei Erkältungskrankheiten und Bauchweh behandeln 85,5\% der Eltern ihre Kinder nach Möglichkeit mit einem pflanzlichen Arzneimittel. Zum Vergleich: In England sind es $28 \%$, in den Niederlanden $13,5 \%$ und in den USA lediglich $10 \%$. Die wichtigste Motivation der Eltern zum Einsatz pflanzlicher Mittel ist entgegen der allgemeinen Annahme - keineswegs die Unzufriedenheit mit der «Schulmedizin», sondern der Wunsch nach einer natürlichen, sicheren und nebenwirkungsarmen Behandlung. Als häufigste Informationsquelle wurde mit $80,2 \%$ der Kinderarzt und nur mit 4,2\% ein Heilpraktiker angegeben. Verglichen mit der «Schulmedizin» bewerteten 13,6\% der Eltern den Erfolg der Behandlung mit pflanzlichen Medikamenten als «grösser», 39,3\% als «gleich gross» und $21,9 \%$ als «kleiner». Der Gesamterfolg der Behandlung mit Phytopharmaka wird von $12 \%$ als «sehr gut», von $55,1 \%$ als «gut», von $26,6 \%$ als «mittel», von $5,1 \%$ als «nicht gut» und lediglich von $1,1 \%$ als «überhaupt nicht gut» eingestuft. Die grosse $\mathrm{Zu}$ friedenheit mit der Phytotherapie zeigt auch folgende Zahl aus der neuen Studie: Bei einer Erkrankung ihres Kindes würden 96,3\% der befragten Eltern erneut zu einem pflanzlichen Arzneimittel greifen. (rfi)

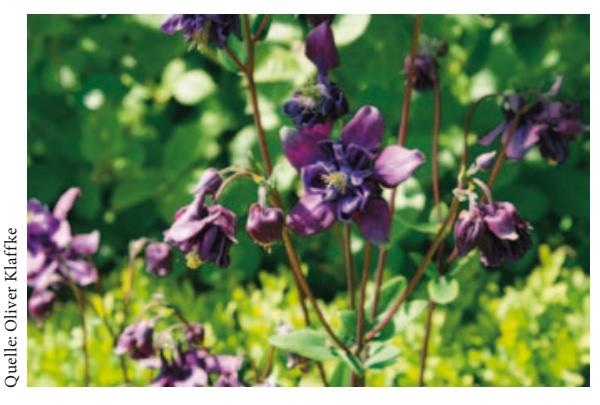

\section{Pharmarichtlinie hilft den Kräuterbauern}

Im solothurnischen Rüttenen ist Anfang Juli die erste computergesteuerte Kräutertrocknungsanlage auf einem Bauernhof der Anbauvereinigung Waldhofkräuter in Betrieb genommen worden. Die damit produzierten Kräuter entsprechen neu den Anforderungen modernster pharmazeutischer Richtlinien. Das erforderliche Know-how zum Bau dieser Trocknungsanlagen haben die Landwirte zusammen mit ihren Kunden erarbeitet. $\mathrm{Zu}$ ihnen gehört seit mehr als 20 Jahren die Pharmafirma PADMA AG. Der Kräuteranbau ist in der Schweizer Landwirtschaft ein Nischenmarkt. Trotz kleiner Absatzmengen hilft er den Bauern, ihr Einkommen neben der Milch- und Viehwirtschaft mit einem zusätzlichen Standbein zu sichern. Die Mitglieder der Anbauvereinigung Waldhofkräuter pflanzen seit über 25 Jahren Kräuter im Grossraum Oberaargau/Emmental an und möchten auch in Zukunft nicht darauf verzichten. Deshalb haben die einzelnen Landwirte in den Bau neuer Trocknungsanlagen investiert. Damit ist gesichert, dass ihre Kräuter den stetig wachsenden Anforderungen von Behörden im In- und Ausland und ihren Kunden entsprechen. Auslöser für die Investitionen war die neue pharmazeutische Richtlinie zur Kräuterherstellung, GACP (Good Agricultural and Collection Practice for Starting Materials of Herbal Origin), die 2006 international in Kraft getreten ist. Um den finanziellen und administrativen Aufwand so gering wie möglich zu halten, die hohen Qualitätsanforderungen der Richtlinie jedoch möglichst präzise umzusetzen, haben sich die Pflanzer mit zwei ihrer Kunden an einen Tisch gesetzt. Gemeinsam mit der Ricola AG, die Hustenbonbons herstellt, und der Arzneimittelherstellerin PADMA AG wurde ein umfassendes Qualitätsdossier erstellt. In diesem Dossier sind die einzelnen Prozesse des Kräutertrocknens genau beschrieben. Bis Ende 2010 sollen die angebauten Kräuter nur noch in Trocknungsanlagen verarbeitet werden, die von den beiden Firmen abgenommenen wurden. Es gilt, alle gesetzlichen Grenzwerte strikt einzuhalten, die etwa bezüglich der Kontamination durch andere Pflanzen sowie durch Schimmel, Aflatoxine oder etwa Mikroorganismen gelten. Die getrockneten Erzeugnisse müssen dabei sowohl den umfangreichen Anforderungen der Lebensmittelbranche als auch den strengen Qualitätsvorschriften der Pharmaindustrie genügen. «Durch die Teamarbeit mit den beiden Firmen konnten unsere Bauern frühzeitig die Weichen für die Zukunft stellen», sagt Markus Daepp, Geschäftsleiter der Anbauvereinigung Waldhofkräuter. In der Kooperation sind rund 50 landwirtschaftliche Betriebe des Grossraums Oberaargau/ Emmental zusammengeschlossen. Sie produzieren jährlich über 50 Tonnen Kräuter. Die langfristige Partnerschaft mit den Abnehmern erlaubt es den Pflanzern, hohe Investitionen, wie die eines Umbaus, zu tätigen. Für die PADMA AG pflanzt die Anbauvereinigung zwei Arten an: Potentilla aurea (Goldfingerkraut) und Aquilegia vulgaris (Gemeine Akelei; siehe Abb.). Akelei ist in der Schweiz eine geschützte Pflanze und darf industriell nur genutzt werden, wenn sie eigens dazu angebaut wird. Für andere Abnehmer, wie z.B. die Ricola AG, werden neben Melisse, Kamille, Pfefferminze und Spitzwegerich insgesamt etwa 30 verschiedene Pflanzen angebaut. (rfi) 